\title{
Estudio retrospectivo sobre concentración de hemoglobina y factores asociados a la anemia posparto en primigestantes menores de 30 años de Lima, Perú, 2010
}

\author{
Oscar Munares-García* \\ Karla Palacios-Rodríguez**
}

\begin{abstract}
*Licenciado en Obstetricia. Magister en Salud Pública. Doctor en Ciencias de la Salud. Profesor Auxiliar Departamento Académico de Obstetricia. Facultad de Medicina Humana San Fernando. Universidad Nacional Mayor de San Marcos. Especialista en Epidemiología Intendencia de Investigación y Desarrollo. Superintendencia Nacional de Salud. Lima. Perú.

**Licenciada en Obstetricia. Especialidad en Promoción de la Salud Materna con mención en Estimulación Prenatal y Psicoprofilaxis Obstétrica. Clínica San Juan de Dios. Lima. Perú.

Correspondencia: Dr. Oscar Munares-García. Av. Grau 1110 Lima 1 Perú. Correo electrónico: omunaresg@unmsm.edu.pe Número telefónico: (051) 961599822
\end{abstract}

\section{RESUMEN}

Introducción: el manejo del parto puede ocasionar consecuencias tanto a la madre como al recién nacido, uno de ellos es la anemia. Objetivo: Determinar la concentración de hemoglobina en el pre y posparto y factores asociados a la anemia posparto en primigestantes menores de 30 años atendidas en un hospital público de Lima, Perú. Materiales y Método: estudio descriptivo retrospectivo en 294 gestantes atendidas para su parto en el Instituto Nacional Materno Perinatal de Lima, Perú. Se consignaron las concentraciones de hemoglobina antes y después del parto, datos sociodemográficos y factores asociados. Resultados: el 67,0\% de las gestantes se encontraban entre los 20 a 29 años. La mediana de hemoglobina antes del parto en las gestantes de parto vaginal fue de 12,5 g/dL y en el posparto fue de 10,6 g/ dL. En las gestantes de parto vaginal el 27,1\% presentó anemia y en el posparto el 56,4\%. En partos por cesárea, el 42,9\% presentó anemia antes del parto y en el posparto el 78,6\%. Los factores que se asociaron fueron hemorragia (ORa: 53,3 IC 95\% 6,6-427,2 p<0,001), trabajo de parto prolongado (ORa: 6,7 IC $95 \%$ 1,5-30,1 $\mathrm{p}=0,013$ ), rotura prematura de membranas (ORa: $2,7 \mathrm{IC} 95 \%$ 1,0-5,5 $\mathrm{p}=0$, 041) y desgarro perineal (ORa: 2,5 IC95\% 1,0-6,1 p=0,041). Conclusiones: existe una reducción de los niveles de hemoglobina de alrededor de $1 \mathrm{~g} / \mathrm{dL}$ en el posparto. Los factores asociados a anemia posparto fueron la hemorragia posparto, trabajo de parto prolongado, rotura prematura de membranas y desgarro perineal. MÉD.UIS. 2017;30(2):37-44.

Palabras clave: Anemia. Periodo Posparto. Hemoglobinas. Embarazo.

\section{Retrospective study on concentration of hemoglobin and factors associated with postpartum anemia in primigestants younger than 30 years old of Lima, Peru, 2010}

\section{ABSTRACT}

Introduction: the management of labor can have consequences for both the mother and the newborn, one of them is anemia. Objective: To determine hemoglobin concentration in pre and postpartum, and factors associated with postpartum anemia in primigravid woman younger than 30 years old who were attended in a public hospital in Lima, Peru. Material and Method: a retrospective, cross-sectional study in 294 postpartum primigravid woman younger than 30 years old attended in the National Institute of Perinatal and Maternal Health in Lima, Peru. Hemoglobin concentrations before and after birth, socio-demographic data and associated factors. Results: $67.0 \%$ of pregnant women were among 20 to 29 years of age. In vaginal delivery, the median hemoglobin before birth was $12.5 \mathrm{~g} / \mathrm{dL}$ and postpartum was $10.6 \mathrm{~g} / \mathrm{dL}$, there was a reduction of $1.9 \mathrm{~g} / \mathrm{dL}$. In vaginal delivery, $27.1 \%$ presented anemia prepartum and $56.4 \%$ postpartum; in cesarean sections, $42.9 \%$ had anemia before delivery and $78.6 \%$ after. The factors that were associated were bleeding $(\mathrm{ORa}: 53.395 \% \mathrm{Cl}$ 6.6-427.2 p<0,001), prolonged labor (ORa: $6.795 \% \mathrm{Cl}$ 1.5-30.1 p=0,013), premature rupture of membranes ORa: 2.7 IC $95 \%$ 1.0-5.5 p=0,041) and perineal tear (ORa: 2.5 IC95\% 1.0-6.1 p=0,041). Conclusions: there is a reduction in hemoglobin levels of around $1 \mathrm{~g} / \mathrm{dL}$ of hemoglobin 
in the postpartum period. Factors associated with postpartum hemorrhage were postpartum haemorrhage, prolonged labor, premature rupture of membranes, and perineal tear. MÉD.UIS. 2017;30(2):37-44.

Keywords: Anemia. Postpartum Period. Hemoglobins. Pregnancy.

¿Cómo citar este artículo?: Munares-García O, Palacios-Rodríguez K. Estudio retrospectivo sobre concentración de hemoglobina y factores asociados a la anemia posparto en primigestantes de Lima, Perú, 2010. MÉD.UIS. 2017;30(2):37-44.

\section{INTRODUCCIÓN}

La anemia durante el embarazo es un problema de salud pública, agudizándose en el tercer trimestre y en el posparto, llegando a casi un cuarto de las gestantes'. El problema de la anemia posparto, ha sido poco estudiado, sobre todo sus factores. La importancia de estudiar la concentración de hemoglobina en el posparto deriva a que si estas concentraciones son muy bajas y no son adecuadamente recuperadas, la madre dará una lactancia materna con pocas reservas de hierro, contribuyendo así a la anemia en el niño, sobre todo en los primeros seis meses de vida que es la recomendación de lactancia materna exclusiva². Durante el parto existe pérdida sanguínea y por ende reducción de los niveles de hemoglobina, este punto se agravaría por las actividades realizadas en la atención del parto como el manejo activo ${ }^{3}$, donde la gestante pierde más sangre, ocasionando así una proporción más alta de anemia en el posparto.

La anemia posparto es un evento con una frecuencia variable, en un estudio realizado por Rivas y colaboradores en México ${ }^{4}$ indicaron que este puede llegar entre un 4 a $27 \%$ de las madres. Romero-Arias y colaboradores, en el 2011 en España, encontraron en 1488 gestantes un $11 \% 5$ de anemia antes del parto, pasando luego a $59 \%$ en el posparto, así mismo en el sur de la India en gestantes evaluadas en dos momentos, a las 36 semanas del embarazo y 6 semanas posparto, se encontró que la anemia fue del $26,8 \%$ y $47,3 \%$ respectivamente ${ }^{6}$, este problema se agudiza más si el parto es por cesárea, pudiendo llegar a requerir una trasfusión sanguínea en el 3,5\%4.

Algunos factores asociados a la aparición de la anemia en el posparto son la edad materna menor a 21 años, inadecuada suplementación con hierro a partir de las 36 semanas, pérdida de sangre percibida por la madre durante el parto y complicaciones en el parto ${ }^{5,6}$.

El objetivo del presente estudio fue determinar las concentraciones de Hemoglobina $(\mathrm{Hb})$ en el pre y posparto y factores asociados a la anemia posparto en primigestantes menores de 30 años atendidas en un hospital de Lima, Perú.

\section{Materiales y método}

Estudio descriptivo, retrospectivo realizado en el Instituto Nacional Materno Perinatal de Lima, Perú, hospital perteneciente al Ministerio de Salud que atiende a gestantes de escasos recursos aseguradas al Seguro Integral de Salud. Se incluyeron primigestantes admitidas de enero a junio de 2010, de parto eutócico o por cesárea, edad menor a 30 años, entre las 23 a 42 semanas de gestación, con resultado de $\mathrm{Hb}$ posparto inmediata ( $\leq 2$ horas), excluyéndose casos con problemas hematológicos (coagulación intravascular diseminada, trombocitopenias, trombofilias), y datos incompletos. Con un $95 \%$ de confianza, a una prevalencia de anemia posparto de $59 \%$ y con $5,4 \%$ de precisión, se obtuvo una muestra de 294 primigestas, se aplicó un muestreo probabilístico proporcional al grupo de edad entre adolescentes y adultas jóvenes.

La información fue solicitada al área de investigación del instituto, el mismo que solicitó los números de historias clínicas de los casos de primigestas adolescentes y adultas jóvenes indicándoles la selección aleatoria y proporcional de las historias, con la historias seleccionadas, se procedió a revisarlas físicamente y registrar las variables en una hoja de registro. Las variables extraídas fueron: edad (años), nivel educativo, estado civil, ocupación, lugar de nacimiento, tipo de parto (vaginal o cesárea), edad gestacional (semanas), macrosomía (peso recién nacido >3999 g), sobrepeso/obesidad (IMC $\left.>25,1 \mathrm{Kg} / \mathrm{m}^{2}\right)$ materna, talla materna baja $(<1,46$ $\mathrm{m})$. Así mismo se registraron el trabajo de parto prolongado (>12 horas), hemorragia posparto (diferencia de $\mathrm{Hb}$ preparto y posparto $\geq 3,2 \mathrm{~g} / \mathrm{dL}$ ) 4,7 , desproporción cefálo-pélvica, rotura prematura de membranas, preeclampsia, y desgarro perineal. Para las concentraciones de $\mathrm{Hb}$ preparto, fueron consideradas muestras tomadas hasta los 120 días 
antes del parto, mientras que para el posparto se emplearon muestras hasta las primeras dos horas después del parto. La anemia se determinó de acuerdo a los parámetros de la Organización Mundial de la Salud ${ }^{8}$ en $\mathrm{Hb}<11 \mathrm{~g} / \mathrm{dL}$.

Los datos corresponden al análisis secundario de datos recolectados de historias clínicas del estudio sobre morbilidad materna en primigestas adolescentes y adultas jóvenes ${ }^{9}$. Se guardó la confidencialidad de los datos y para el análisis no se registró identificación, como nombres o número de historia clínica; trabajando los datos a través de códigos, por lo que la información se consideró anónima.

Los datos fueron analizados en una base de datos en SPSS v20.0. Se aplicaron distribución de frecuencias y porcentajes en las variables cualitativas. En las variables cuantitativas se aplicó la media, mínimo y máximo. Para la determinación de las diferencias entre las concentraciones de hemoglobina preparto y posparto, se aplicó la prueba de Wilcoxon y Chi cuadrado para la determinación de las diferencias significativas y factores asociados a la anemia posparto, todos a un nivel de significación estadístico $\mathrm{p}<0,05$. Para la determinación de los factores asociados, a las variables significativas con la prueba de Chi cuadrado, se aplicó la razón de productos cruzados (OR) con sus intervalos de confianza al 95\%, para la determinación de asociaciones crudas, finalmente para la determinación del modelo explicativo de la anemia posparto, (asociaciones ajustadas u ORa) se aplicó regresión logística binaria bajo el método introducir (procedimiento donde las variables seleccionadas se introducen en un solo bloque) y para su determinación significativa se empleó los intervalos de confianza al 95\%. Para la determinación de la proporción de la varianza explicada de los factores asociados en la regresión, se empleó el $\mathrm{R}^{2}$ Cox y Snell, para la determinación de la bondad de ajuste del modelo se aplicó la prueba de Hosmer y Lemeshow, considerándose un modelo adecuado cuando el valor $\mathrm{p}>0,05$. Para la determinación de la predictibilidad de la concentración de hemoglobina preparto para no presentar anemia posparto, se aplicó la curva ROC.

\section{Resultados}

Durante el periodo estudiado, hubo 4064 casos de primigestas, utilizándose 294 para el estudio. El 67,0\% se encontraban entre los 20 a 29 años, 76,9\% tenía educación secundaria, $66,7 \%$ era conviviente, $83,3 \%$ era ama de casa y 60,9\% nació en Lima, la capital de la república (Ver Tabla 1). La mediana de edad fue 21 años, con un rango de 13 a 29 años y la de edad gestacional promedio fue de 39 semanas, con un rango de 23 a 42 semanas (Ver Tabla 2).

Tabla 1. Características generales de las gestantes atendidas Instituto Nacional Materno Perinatal.

\begin{tabular}{|c|c|c|}
\hline & $\mathbf{n}$ & $\%$ \\
\hline \multicolumn{3}{|c|}{ Edad (años) } \\
\hline 13 a 19 & 97 & 33 \\
\hline 20 a 29 & 197 & 67 \\
\hline \multicolumn{3}{|c|}{ Nivel educativo } \\
\hline Primaria & 29 & 9,9 \\
\hline Secundaria & 226 & 76,9 \\
\hline Superior & 39 & 13,3 \\
\hline \multicolumn{3}{|c|}{ Estado civil } \\
\hline Casada & 19 & 6,5 \\
\hline Soltera & 78 & 26,5 \\
\hline Conviviente & 196 & 66,7 \\
\hline Otra & 1 & 0,3 \\
\hline \multicolumn{3}{|c|}{ Ocupación } \\
\hline Ama de casa & 245 & 83,3 \\
\hline Estudiante & 21 & 7,1 \\
\hline Otro & 28 & 9,5 \\
\hline \multicolumn{3}{|c|}{ Lugar de nacimiento } \\
\hline Lima & 179 & 60,9 \\
\hline Provincia & 115 & 39,1 \\
\hline Total & 294 & 100,0 \\
\hline
\end{tabular}

Fuente: Autor

La mediana de la diferencia de $\mathrm{Hb}$ fue mayor en las mujeres de parto por cesárea $(1,5 \mathrm{~g} / \mathrm{dL})$ que las de parto vaginal $(0,7 \mathrm{~g} / \mathrm{dL})$. En mujeres de parto vaginal se encontraron diferencias significativa en la mediana de $\mathrm{Hb}$ preparto y posparto $(\mathrm{p}<0,001)$, así como en mujeres de parto por cesárea $(p<0,001)$ (Ver Tabla 2).

En general, el 27,9\% de las gestantes presentó anemia preparto, y en el posparto llegó a 57,5\%, las diferencias fueron significativas para el parto vaginal y la población en general (Ver Figura 1). En las gestantes de parto vaginal, el 27,1\% presentó anemia en el preparto y 56,4\% en el posparto, es decir, hubo un incremento de 29,3 puntos porcentuales; en gestantes de parto por cesárea, el 42,9\% presentó anemia preparto, y $78,6 \%$ en el posparto. Cabe 
resaltar que todas las gestantes que tuvieron anemia preparto, presentaron anemia posparto (OR: 2,47 IC95\% 2,07:2,86 p<0,001).

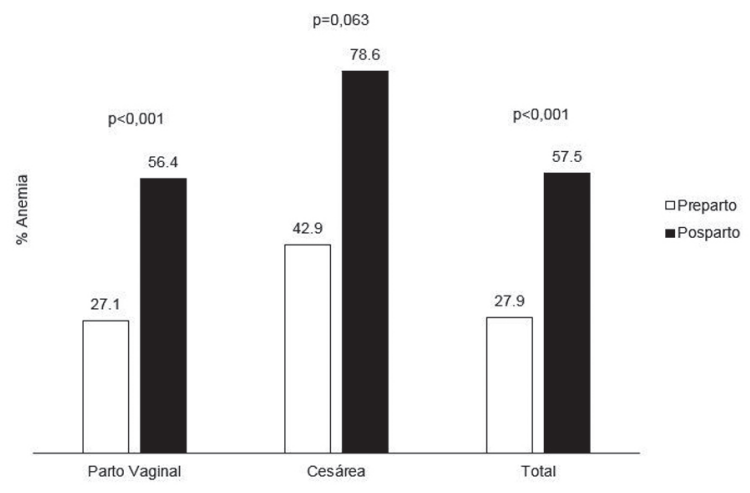

Figura 1. Proporción de anemia $(\mathrm{Hb}<11,0 \mathrm{~g} / \mathrm{dl})$ antes y después del parto según tipo de parto

Fuente: autor

Tabla 2. Estadísticos descriptivos de los niveles de hemoglobina (g/dl) según momento y tipo de parto, Instituto Nacional Materno Perinatal

\begin{tabular}{|c|c|c|c|c|c|}
\hline & $\mathbf{n}$ & Mediana & Min & Máx & $\mathbf{p}$ \\
\hline Edad (años) & 294 & 21 & 13 & 29,0 & \\
\hline $\begin{array}{l}\text { Edad gestacional } \\
\text { (sem) }\end{array}$ & 294 & 39 & 23 & 42,0 & \\
\hline \multicolumn{6}{|l|}{ Parto vaginal } \\
\hline $\mathrm{Hb}$ Preparto & 280 & 11,7 & 7,6 & 16,4 & \\
\hline Hb Posparto & 280 & 10,6 & 4,4 & 14,0 & $<0,001$ \\
\hline Diferencia & 280 & 0,7 & 0,0 & 5,2 & \\
\hline \multicolumn{6}{|l|}{ Cesárea } \\
\hline $\begin{array}{l}\text { Hb Preparto (g/ } \\
\text { dl) }\end{array}$ & 14 & 11,1 & 8,5 & 13,1 & \\
\hline $\begin{array}{l}\text { Hb Posparto (g/ } \\
\text { dl) }\end{array}$ & 14 & 9,6 & 6,1 & 11,4 & $<0,001$ \\
\hline Diferencia & 14 & 1,5 & 0,3 & 3,9 & \\
\hline \multicolumn{6}{|l|}{ Total } \\
\hline $\begin{array}{l}\text { Hb Preparto (g/ } \\
\text { dl) }\end{array}$ & 294 & 11,6 & 7,6 & 16,4 & \\
\hline $\begin{array}{l}\text { Hb Posparto (g/ } \\
\text { dl) }\end{array}$ & 294 & 10,5 & 4,4 & 14,0 & $<0,001$ \\
\hline Diferencia & 294 & 0,7 & 0,0 & 5,2 & \\
\hline
\end{tabular}

Fuente: Autor

En el análisis bivariado, no se encontró asociación con la rotura prematura de las membranas, desproporción céfalo-pélvica, desgarro perineal, preeclampsia, macrosomía, sobrepeso/obesidad, parto pretérmino, terminación del parto en cesárea, talla materna baja y la anemia posparto. Por otro lado se encontró asociación con hemorragia posparto (OR: 32,4 IC95\% 4,4-240,0), trabajo de parto prolongado (OR: 2,8 IC95\% 1,1-7,2) y ser adolescente (OR: 1,7 IC $95 \%$ 1,0-2,8). Mientras que con el modelo para la determinación de factores asociados a la anemia posparto se asoció la hemorragia posparto (ORa: 53,2 IC 95\% 6,6-427,2), trabajo de parto prolongado (ORa: 6,7 IC95\% 1,5-30,1), rotura prematura de las membranas (ORa: 2,4 IC 95\% 1,0-5,5) y desgarro perineal (ORa: 2,5 IC95\% 1,0-6,1). El resultado de la prueba de Hosmer y Lemeshow fue $p=0,744$ indicando que la evaluación del ajuste global del modelo fue adecuado; la prueba de ómnibus resultó significativa $(p<0,001)$ indicando que el modelo ayuda a explicar el fenómeno de la anemia posparto. El porcentaje global del modelo de regresión logística binario, que es la manera en que coincide el valor pronosticado con el valor observado fue de $65,3 \%$ por lo que el modelo clasifica correctamente las variables predictoras. De las doce variables propuestas como asociadas a la anemia posparto en el análisis bivariado, se ajustaron a través de la regresión logística binaria, quedando como variables predictoras la hemorragia posparto, el trabajo de parto prolongado, la rotura prematura de membranas y el desgarro perineal, este conjunto de variables lograron explicar entre el 16,8 al $22,5 \%$ de la varianza de la anemia posparto $\left(\mathrm{R}^{2}\right.$ Cox Snell= $0,168 R^{2}$ Nagelkerke= 0,225) (Ver Tabla 3).

Finalmente se encontró en partos vaginales que el tener una concentración de $\mathrm{Hb}$ preparto de 11,75 g/ dl o más predice no tener anemia en el posparto con un $86,9 \%$ de sensibilidad y $79,7 \%$ de especificidad, la curva ROC nos indicó que el poder predictivo es adecuado, con un área bajo la curva de 0,872 , con intervalos de confianza estadísticamente significativos $(p<0,001)$ (Ver Figura 2).
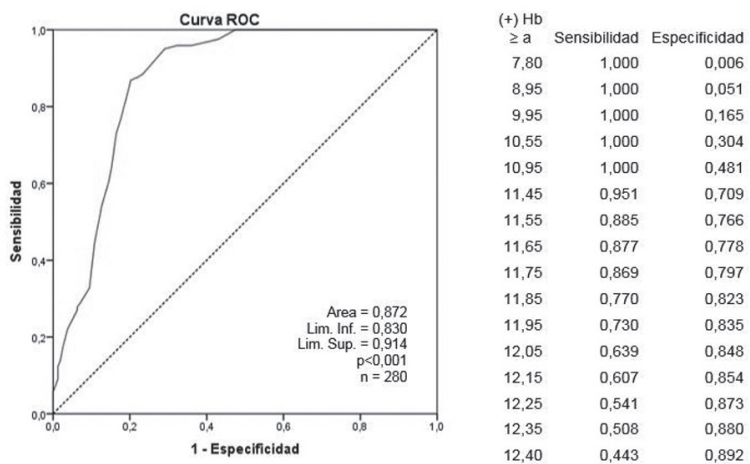

Figura 2. Curva ROC, para predicción de no presentar anemia posparto en función de la $\mathrm{Hb}$.

Fuente: autor 
Tabla 3. Factores asociados a la anemia posparto Instituto Nacional Materno Perinatal, Lima Perú

\begin{tabular}{|c|c|c|c|c|}
\hline & \multicolumn{2}{|c|}{ Anemia posparto } & \multirow{2}{*}{$\begin{array}{l}\text { OR crudo } \\
\text { (IC95\%) }\end{array}$} & \multirow{2}{*}{$\begin{array}{c}\text { OR } \\
\text { ajustado } \\
\text { (IC95\%) }\end{array}$} \\
\hline & $\mathrm{Si}$ & No & & \\
\hline & $n=169$ & $n=125$ & & \\
\hline & $\%$ & $\%$ & & \\
\hline Hemorragia posparto & & & $p<0,001$ & $p<0,001$ \\
\hline $\mathrm{Si}$ & 20,7 & 0,8 & $\begin{array}{c}32,4 \\
(4,4: 240)\end{array}$ & $\begin{array}{c}53,2 \\
(6,6: 427,2)\end{array}$ \\
\hline No & 79,3 & 99,2 & 1 & 1 \\
\hline $\begin{array}{l}\text { Trabajo de parto } \\
\text { prolongado }\end{array}$ & & & $p=0,025$ & $p=0,013$ \\
\hline $\mathrm{Si}$ & 12,4 & 4,8 & $\begin{array}{c}2,8 \\
(1,1: 7,2)\end{array}$ & $\begin{array}{c}6,7 \\
(1,5: 30,1)\end{array}$ \\
\hline No & 87,6 & 95,2 & 1 & 1 \\
\hline $\begin{array}{l}\text { Rotura prematura de } \\
\text { membranas }\end{array}$ & & & $p=0,578$ & $p=0,041$ \\
\hline $\mathrm{Si}$ & 26 & 23,2 & $\begin{array}{c}1,2 \\
(0,7: 2,0)\end{array}$ & $2,4(1,0: 5,5)$ \\
\hline No & 74 & 76,8 & 1 & 1 \\
\hline $\begin{array}{l}\text { Desproporción } \\
\text { cefálo-pélvica }\end{array}$ & & & $p=0,626$ & $p=0,138$ \\
\hline $\mathrm{Si}$ & 14,8 & 12,8 & $\begin{array}{c}1,2 \\
(0,6: 2,3)\end{array}$ & $2,1(0,8: 5,4)$ \\
\hline No & 85,2 & 87,2 & 1 & 1 \\
\hline Desgarro Perineal & & & $p=0,323$ & $p=0,041$ \\
\hline $\mathrm{Si}$ & 39,1 & 44,8 & $\begin{array}{c}0,8 \\
(0,5: 1,3)\end{array}$ & $2,5(1,0: 6,1)$ \\
\hline No & 60,9 & 55,2 & 1 & 1 \\
\hline Preeclampsia & & & $p=0,650$ & $p=0,102$ \\
\hline $\mathrm{Si}$ & 11,2 & 9,6 & $\begin{array}{c}1,2 \\
(0,6: 2,6)\end{array}$ & $2,2(0,9: 5,9)$ \\
\hline No & 88,8 & 90,4 & 1 & 1 \\
\hline Adolescente & & & $p=0,039$ & $p=0,117$ \\
\hline $\mathrm{Si}$ & 37,9 & 26,4 & $\begin{array}{c}1,7 \\
(1,0: 2,8)\end{array}$ & $1,6(0,9: 2,7)$ \\
\hline No & 62,1 & 73,6 & 1 & 1 \\
\hline Macrosomía & & & $p=0,408$ & $p=0,506$ \\
\hline $\mathrm{Si}$ & 5,9 & 3,2 & $\begin{array}{c}1,9 \\
(0,6: 6,2)\end{array}$ & $1,6(0,4: 5,7)$ \\
\hline No & 94,1 & 96,8 & 1 & 1 \\
\hline Sobrepeso/obesidad & & & $p=0,860$ & $p=0,588$ \\
\hline $\mathrm{Si}$ & 68,6 & 69,6 & $\begin{array}{c}1,0 \\
(0,6: 1,6)\end{array}$ & $1,2(0,7: 2,1)$ \\
\hline No & 31,4 & 30,4 & 1 & 1 \\
\hline Parto Pretérmino & & & $p=0,098$ & $p=0,983$ \\
\hline $\mathrm{Si}$ & 7,7 & 13,6 & $\begin{array}{c}0,5 \\
(0,2: 1,1)\end{array}$ & $1,0(0,4: 2,6)$ \\
\hline No & 92,3 & 86,4 & 1 & 1 \\
\hline
\end{tabular}

\begin{tabular}{|l|c|c|c|c|}
\hline & \multicolumn{2}{|c|}{ Anemia posparto } & $\begin{array}{c}\text { OR crudo } \\
\text { (IC95\%) }\end{array}$ & $\begin{array}{c}\text { OR } \\
\text { ajustado } \\
\text { (IC95\%) }\end{array}$ \\
\hline Término del parto & & & $p=0,164$ & $p=0,969$ \\
\hline Cesárea & 6,5 & 2,4 & $\begin{array}{c}2,8 \\
(0,8: 10,4)\end{array}$ & $1,0(0,2: 6,8)$ \\
\hline Vaginal & 93,5 & 97,6 & 1 & 1 \\
\hline $\begin{array}{l}\text { Talla materna baja } \\
(<\mathbf{1 , 4 6} \mathbf{m})\end{array}$ & & & $p=0,228$ & $p=0,441$ \\
\hline Si & 6,5 & 10,4 & 0,6 & $0,7(0,3: 1,7)$ \\
\hline No & 93,5 & 89,6 & 1 & 1 \\
\hline Total & 100 & 100 & & \\
\hline
\end{tabular}

R2 Cox Snell $=0,168$ R2 Nagelkerke $=0,225$ Prueba de Hosmer $y$ Lemeshow $p=0,744$ Porcentaje global $=65,3$ Prueba de ómnibus $(p<0,001)$

Fuente: Autor

\section{Discusión}

El sangrado es un fenómeno fisiológico inherente al parto y puerperio, con respecto al parto vaginal, el estudio encontró que se pierde alrededor de $1 \mathrm{~g} /$ $\mathrm{dL}$ de $\mathrm{Hb}$ y casi $2 \mathrm{~g} / \mathrm{dL}$ cuando es por cesárea (Ver Tabla 2), la proporción de anemia en el posparto se duplica comparada con el preparto (Ver Figura 1). Fisiológicamente se ha determinado que alrededor de las seis semanas posparto, la $\mathrm{Hb}$ y el hematocrito regresan a sus niveles previos en ausencia de una pérdida sanguínea excesiva, (definida esta última cuando se presenta una diferencia en las concentraciones de $\mathrm{Hb}$ pre y posparto $\geq 3,5 \mathrm{~g} / \mathrm{dL})^{5}$ y se sabe que la estimación clínica de la pérdida sanguínea es sobrestimada ${ }^{10}$, pero este postulado es correcto siempre y cuando se tengan adecuadas reservas de hierro ${ }^{11}$, lo que probablemente resulte difícil con muchas pacientes que ya son anémicas. El paso a seguir luego del parto sería prescribirle a las puérperas con anemia sales de hierro para recuperar sus niveles de $\mathrm{Hb}$ y así poder sostener una lactancia materna con adecuadas concentraciones de hierro para el recién nacido aunque la evidencia muestra que son pocas las mujeres que consumen hierro posterior al parto. Monárrez-Espino y col., $(2001)^{12}$ identificaron en su estudio de mujeres en diferentes periodos reproductivos, que la proporción de anemia se incrementa durante la lactancia materna, e incluso es mayor que en los trimestres previos al parto, quizá ello explique elevadas prevalencias de anemia en niños entre los 6 a 59 meses (32,6\%), mayor aun entre los 6 y 8 meses, llegando a $59,4 \%{ }^{13}$. 
La anemia posparto es un evento frecuente, el estudio encontró que un 56,4\% de casos lo presentaron (57,5\% en parto vaginal y $78,6 \%$ en parto por cesárea). En Colombia, Holguín-Hernández y Orozco-Díaz (2012) ${ }^{14}$ encontraron una prevalencia de 7,6\%, Romero-Arias et al, (2011) 5 en España llegó al 59,0\%, Manjuanth col., $(2014)^{6}$ en India identificó $47,3 \%$ y en Alemania llegó al 22\%15. Este evento puede mantenerse por más tiempo, Monárrez-Espino y col., (2001) ${ }^{12}$ en México encontraron $42,9 \%$ de anemia hasta los primeros seis meses después del parto.

Un elemento importante a determinar es lo correspondiente a la concentración de $\mathrm{Hb}$ antes del parto, el estudio determinó que si las concentraciones deHbson mayoresa11,75g/dL, existe una probabilidad menor de no presentar anemia posparto, con una sensibilidad del $86,9 \%$ y una especificidad del $79,7 \%$ (Ver Figura 2); esto pone de manifiesto que uno de los objetivos primordiales en controles prenatales es asegurar que las concentraciones en la gestante lleguen al tercer trimestre con cifras mayores a $12 \mathrm{~g} /$ dL; esto también indicaría fallas en la suplementación con hierro que presentan las gestantes al término del embarazo, con inapropiados consejos nutricionales, aunado a los problemas gastrointestinales propios del crecimiento uterino, lo que hace que esta estrategia tenga que replantearse.

El estudio encontró además, que la hemorragia posparto, el trabajo de parto prolongado, la rotura prematura de las membranas y el desgarro perineal se asociaron a anemia posparto. En India se identificó a la anemia a partir de las 36 semanas, la pérdida de sangre percibida por la madre durante el parto, edad materna menor a 21 años y la administración inadecuada de suplementos de hierro asociadas a la anemia posparto ${ }^{6}$. Por otra parte, un estudio realizado en Tanzania encontró que la suplementación de hierro y ácido fólico es un factor protector de complicaciones en el parto ${ }^{16}$. Otro estudio realizado en India, aunque no fue realizado en primigestas, encontró que el no tener nivel educativo, paridad menor o igual a dos, intervalo intergenésico menor o igual a tres años, anemia en el primer o segundo trimestre se asociaron con la anemia a las seis semanas después del parto ${ }^{15}$.

Es evidente que la pérdida excesiva de sangre durante el parto resulta en una reducción de las concentraciones de hemoglobina posparto, esto es más evidente en la hemorragia posparto. Mediante una técnica de dilución de hemoglobina, Wallace (citado por Rubio-Romero y col., 2008) estimó la magnitud del sangrado en cerca de $450 \mathrm{~mL}$ y consideró que el 6 a $17 \%$ de las mujeres presentan un sangrado mayor de $500 \mathrm{~mL}^{17}$. Otro elemento que también produce pérdida de sangre, pero en menor medida que la hemorragia posparto es lo correspondiente al desgarro perineal, sobre todo los desgarros tipo $203^{11}$.

El parto prolongado, es un fenómeno donde los tiempos para el proceso del parto, se incrementan a periodos mayores que los previstos fisiológicamente. Resulta interesante encontrar una asociación entre el parto prolongado y la anemia posparto, este punto podría deberse a que luego del parto el músculo uterino debe pasar al proceso de involución y por ende la herida que deja el desprendimiento de la placenta en el útero no sería adecuadamente cerrada en gestantes que presentan parto prolongado por cansancio de las fibras musculares, ello puede ser más dramático con el empleo de la inducción o acentuación con oxcitocina, puesto que $4 \%$ de las gestantes que reciben oxcitocina sufren de hemorragia posparto ${ }^{18}$.

La rotura prematura de las membranas, no ha sido asociada anteriormente con la anemia posparto. Este mecanismo resulta de una malformación en las membranas corioamnióticas que producirían su desprendimiento ${ }^{18}$, como se sabe, el manejo de este evento se basa en el empleo de antibióticos y la terminación del parto, generalmente por inducción o acentuación, ello puede provocar una sobre estimulación del músculo uterino, favoreciendo así un aumento del sangrado en el puerperio y disminución de los niveles de hemoglobina en el posparto.

El presente estudio no encontró asociación con preeclampsia, adolescencia, macrosomía, sobrepesoobesidad, parto pretérmino, cesárea o talla materna baja. Con respecto a la preeclampsia, HolguínHernández y Orozco-Díaz (2012) ${ }^{14}$ no encontraron diferencias significativas entre la anemia y la preeclampsia, pero si encontraron diferencias con la hipertensión. Con respecto a la adolescencia, Palacios ${ }^{9}$ determinó $17,5 \%$ de hemorragia posparto en gestantes adolescentes, encontrado una asociación 1,9 veces mayor a diferencia de la adulta joven (OR: 1,99 IC95\% 0,98-4,03) y Rakesh et al encontraron asociación entre la edad menor a 21 años y la anemia seis meses después del parto (OR: 2,93 IC95\% 1,117,77 ), Bergmann y et al. ${ }^{19}$ determinaron que el grupo adolescente presentó anemia en posparto en un 
4,6\%, encontrando que el no ser adolescente reduce la probabilidad de anemia posparto. Harsha y et al. ${ }^{15}$ no pudieron encontrar asociación entre edad menor o igual de 20 años y anemia en el posparto, Romero-Arias y et al. ${ }^{5}$ no pudieron determinar que la edad mayor a 35 años sea un factor para sangrado excesivo en el parto vaginal.

Con respecto a la macrosomía, este estudio encontró una proporción del 5,9\% con anemia posparto, similar a lo encontrado por Bergmann et al. ${ }^{22}$ donde fue del 4,6\%, sin embargo, en este último sí se encontró asociación entre la macrosomía y la anemia posparto (OR: 1,8 p=0,001). Romero-Arias et al5 no encontraron asociación entre pérdida hemática excesiva en el parto (vaginal o cesárea) con recién nacidos <4000 g. En lo que corresponde al parto pretérmino, este estudio encontró una proporción de 7,7\%, mientras que Bergmann et al. ${ }^{22}$ encontraron una proporción de $4,5 \%$, pero, al igual que como ocurrió en este estudio, no se encontró asociación con anemia posparto. En cuanto a la cesárea se presentó un $6,5 \%$ de casos que terminaron en anemia posparto, Bergmann et al. ${ }^{22}$ encontraron una proporción de $4,5 \%$ en cesárea electiva y de $5,1 \%$ en cesáreas de emergencia, pero con la diferencia de que ellos si encontraron una asociación (OR: 1,88 p<0,001 para cesárea electiva y OR: 2,05 p<0,001 para cesáreas de emergencia). Holguín-Hernández y Orozco-Díaz $(2012)^{14}$ encontraron diferencias significativas entre la anemia y la cesárea, siendo de $28,3 \%$ en las anémicas y de $18,9 \%$ en las no anémicas.

Quizá uno de los puntos, que hay que traer a reflexión, son las estrategias para mejorar la concentración de hemoglobina en este grupo de pacientes, aquellas que terminan con anemia posparto provienen de una dieta pobre en hierro ${ }^{20}$, y este problema presentará una producción de leche disminuida ${ }^{21}$ y lo que se ha podido demostrar es que si se emplean esquemas educativos de la enseñanza de alimentos ricos en hierro en el posparto mejoraría de manera más apropiada el consumo del mismo ${ }^{22}$, aunado también a suplementos de hierro posparto, con las mismas salvedades que en el preparto y los mismos efectos adversos a informar a la paciente.

Existen pocos estudios que hayan tratado de determinar los factores asociados a la anemia posparto 6,15 , por lo que lo presentado contribuye a esclarecer este evento en la región estudiada, pero al ser un estudio basado en historias clínicas no se pudo estudiar otros factores o determinar asociaciones con factores identificados en otros estudios como la edad en mayores de 30 años, el nivel educativo, número de embarazos, controles prenatales menor a 3, consumo de hierro y ácido fólico durante el embarazo $^{6}$ ello podría deberse al tamaño de la muestra y al diseño empleado.

\section{CONCLUSIONES}

Existe una reducción de los niveles de hemoglobina después del parto, siendo en promedio de 1,2 g/dL para el parto vaginal, 1,6 g/dL en el parto por cesárea y en general 1,2 g/dL. La proporción de anemia aumentó en general siendo antes del parto de 27,9\% a $57,5 \%$ en el posparto, siendo más marcado en los partos por cesárea que los partos vaginales. Los factores asociados a la anemia posparto fueron la hemorragia posparto, trabajo de parto prolongado, rotura prematura de membranas y desgarro perineal.

\section{FinANCIACIÓN}

El estudio fue autofinanciado.

\section{CONFLICTO DE INTERESES}

Los autores declaran no tener conflicto de intereses.

\section{Consideraciones Éticas}

Estudiado y aprobado por el Comité de Ética del Instituto Nacional Materno Perinatal: Expediente $\mathrm{N}^{\circ}$ 005852-1.

\section{Referencias Biblograficas}

1. Gómez-Sánchez I, Rosales S, Agreda L, Castillo A, AlarcónMatutti E, Gutiérrez C. Nivel de hemoglobina y prevalencia de anemia en gestantes según caracteristicas socio-demográficas y perinatales. REV. PERU. EPIDEMIOL. 2014;18(2):1-6.

2. Urquizo Aréstegui R. Lactancia materna exclusiva ¿siempre? Rev Per Ginecol Obs. 2014:60(2);171-6.

3. Echevarría Restrepo LG, Campo Campo MN, Gómez Mejía AC. Manejo activo del trabajo de parto y su relación con la morbilidad materno perinatal. Rev Colomb Obs. 2004;55(2): 122-8.

4. Llamas Rivas R, López López E, Gastélum Parra C. Hemorragia en obstetricia. Rev Mex Med Tran. 2010:3 Supl 1:14-20.

5. Romero-Arias AI, Luján-Prior M, Pernia-Fernández J, HernándezMartínez. Incidencia y factores relacionados con la pérdida hemática intraparto excesiva. Enferm Clín. 2011;21: 256-63.

6. Rakesh PS, Gopichandran V, Jamkhandi D, Manjunath K, George K, Prasad J. Determinants of postpartum anemia among women from a rural population in southern India. Int J Womens Heal. 2014:6;395-400.

7. Vizarreta L, Romero RA, Salazar GJ, Lévano A, Saona P. Correlación entre la pérdida sanguínea estimada y la pérdida sanguínea calculada, en partos vaginales en nulíparas. Rev Per 
Ginecol Obstet. 2010:56;155-60.

8. Mejía ME, Quintero VH, Tovar MC. ¿La episiotomía debe ser un procedimiento de rutina?. Colomb Med [Internet]. 2004 [Citado 7 Jun 2017];35(2):75-81. Disponible en: http://www.uacm.kirj. redalyc.redalyc.org/articulo.oa?id $=28335205$

9. Palacios-Rodriguez KP. Morbilidad materna en primigestas adolescentes y adultas jóvenes, Instituto Nacional Materno Perinatal, 2010 [Tesis]. Lima: Cybertesis UNMSM; 2012

10. Breymann C. Tratamiento de la anemia por deficiencia de hierro en el embarazo y en el posparto. Rev Per Ginecol Obstet. 2012;58(4):313-28

11. Espitia F, Orozco L. Anemia en el embarazo, un problema de salud que puede prevenirse. MED.UIS. 2013;26(3):45-50

12. Monárrez-Espino J, Martínez H, Greiner T. Iron deficiency anemia in Tarahumara women of reproductive-age in Northern Mexico. Salud Pública Méx. 2001;43(5):392-401

13. Instituto Nacional de Estadística e Informática. Perú Encuesta Demográfica y de Salud Familiar 2015 Nacional y Departamental. (2016).

14. Holguín-Hernández E, Orozco-Diaz J. Uso de hierro en forma de medicamento en gestantes colombianas. Rev salud pública. 2012;14(5):789-801

15. Harsa Kumar HN, Gupta S, Ruhela S, Tanya S. A Retrospective Study on Magnitude and Factors Associated with Anemia in Postnatal Period from Coastal South India. Ann Med Health Sci Res. 2014:4(5):775-9.
16. Kavle JA, Stoltzfus RJ, Witter F, Tielsch JM, Khalfan SS, Caulfield LE. Association between anaemia during pregnancy and blood loss at and after delivery among women with vaginal births in Pemba Island, Zanzibar, Tanzania. J Health Popul Nutr. 2008;26(2):232-40.

17. Rubio-Romero JA, Gaitán-Duarte HG, Rodríguez-Malagón N. Concordancia entre la estimación visual y la medición del volumen recolectado en una bolsa del sangrado intraparto en mujeres con parto normal en Bogotá, Colombia, 2006. Rev Colomb Obstet Ginecol. 2008;59(2):92-102.

18. Rivera R, Caba F, Smirnow M, Aguilera J, Larraín A. Fisiopatologia de la rotura prematura de las membranas ovulares en embarazos de pretérmino. Rev Chil Obstet Ginecol. 2004:69(3):249-255.

19. Bergmann RL, Richter R, Bergmann KE, Dudenhausen JW Prevalence and risk factors for early postpartum anemia. Eur J Obstet Gynecol Reprod Biol. 2010;150(2):126-31.

20. Estrata, D. Hábitos alimentarios y factores culturales en mujeres embarazadas que acuden a consulta externa del hospital básico Dr. Eduardo Montenegro del Canton Chillanes, provincia de Bolivar, 2010. (Escuela Superior Politecnica de Chimborazo, 2010).

21. Ciudad A. Requerimiento de micronutrientes y oligoelementos. Rev Peru Ginecol Obstet. 2014;60(2):161-70.

22. Cardero Y, Sarmiento R, Selva A. Importancia del consumo de hierro y vitamina C para la prevención de anemia ferropénica. MEDISAN. 2009;13(6):1-13. 